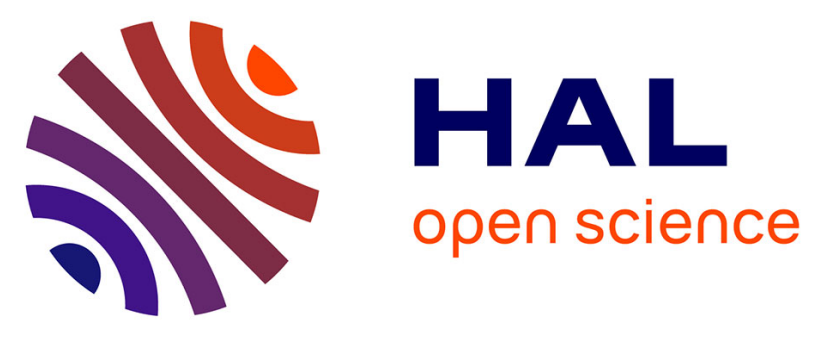

\title{
Oxygen Ion Energization at Mars: Comparison of MAVEN and Mars Express Observations to Global Hybrid Simulation
}

Riku Järvinen, David A. Brain, Ronan Modolo, Andrei Fedorov, Mats Holmström

\section{To cite this version:}

Riku Järvinen, David A. Brain, Ronan Modolo, Andrei Fedorov, Mats Holmström. Oxygen Ion Energization at Mars: Comparison of MAVEN and Mars Express Observations to Global Hybrid Simulation. Journal of Geophysical Research Space Physics, 2018, 123 (2), pp.1678 - 1689. 10.1002/2017JA024884 . insu-01740806

\section{HAL Id: insu-01740806 https://hal-insu.archives-ouvertes.fr/insu-01740806}

Submitted on 27 Aug 2020

HAL is a multi-disciplinary open access archive for the deposit and dissemination of scientific research documents, whether they are published or not. The documents may come from teaching and research institutions in France or abroad, or from public or private research centers.
L'archive ouverte pluridisciplinaire HAL, est destinée au dépôt et à la diffusion de documents scientifiques de niveau recherche, publiés ou non, émanant des établissements d'enseignement et de recherche français ou étrangers, des laboratoires publics ou privés. 


\section{Journal of Geophysical Research: Space Physics}

\section{RESEARCH ARTICLE \\ 10.1002/2017JA024884 \\ Special Section: \\ Oxygen lon Energization at Mars: Comparison of MAVEN and Mars Express Observations to Global Hybrid Simulation}

Mars Aeronomy

Key Points:

- Mars heavy ion plume is measured by multispacecraft observations

- Ion time and distance of flight are estimated based on a global model

- Oxygen ions are observed near Mars within the first half of their gyrophase

\section{Correspondence to:}

R. Jarvinen,

riku.jarvinen@fmi.fi

\section{Citation:}

Jarvinen, R., Brain, D. A., Modolo, R.,

Fedorov, A., \& Holmström, M.

(2018). Oxygen ion energization

at Mars: Comparison of MAVEN

and Mars express observations

to global hybrid simulation.

Journal of Geophysical Research:

Space Physics, 123, 1678-1689.

https://doi.org/10.1002/2017JA024884

Received 13 OCT 2017

Accepted 27 JAN 2018

Accepted article online 8 FEB 2018

Published online 27 FEB 2018

○2018. American Geophysical Union. All Rights Reserved.

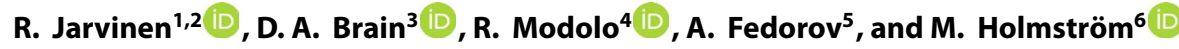 \\ ${ }^{1}$ Finnish Meteorological Institute, Helsinki, Finland, ${ }^{2}$ Department of Electronics and Nanoengineering, School of Electrical \\ Engineering, Aalto University, Espoo, Finland, ${ }^{3}$ Laboratory for Atmospheric and Space Physics, University of Colorado \\ Boulder, Boulder, CO, USA, ${ }^{4}$ LATMOS/IPSL, UVSQ Université Paris-Saclay, UPMC University Paris CNRS, Guyancourt, France, \\ ${ }^{5}$ Université de Toulouse, UPS-OMP, IRAP, Toulouse, France, ${ }^{6}$ Swedish Institute of Space Physics, Kiruna, Sweden
}

Abstract We study oxygen ion energization in the Mars-solar wind interaction by comparing particle and magnetic field observations on the Mars Atmosphere and Volatile EvolutioN (MAVEN) and Mars Express missions to a global hybrid simulation. We find that large-scale structures of the Martian-induced magnetosphere and plasma environment as well as the Mars heavy ion plume as seen by multispacecraft observations are reproduced by the model. Using the simulation, we estimate the dynamics of escaping oxygen ions by analyzing their distance and time of flight as a function of the gained kinetic energy along spacecraft trajectories. In the upstream region the heavy ion energization resembles single-particle solar wind ion pickup acceleration as expected, while within the induced magnetosphere the energization displays other features including the heavy ion plume from the ionosphere. Oxygen ions take up to $80 \mathrm{~s}$ and travel the distance of $20,000 \mathrm{~km}$ after their emission from the ionosphere to the induced magnetosphere or photoionization from the neutral exosphere before they have reached energies of $10 \mathrm{keV}$ in the plume along the analyzed spacecraft orbits. Lower oxygen ion energies of $100 \mathrm{eV}$ are reached faster in 10-20 s over the distance of 100-200 km in the plume. Our finding suggests that oxygen ions are typically observed within the first half of their gyrophase if the spacecraft periapsis is on the hemisphere where the solar wind convection electric field points away from Mars.

\section{Introduction}

Mars is the second smallest object in the solar system with a significant atmosphere. The planet has likely lost a lot, even an oceanful, of water since its childhood. As $89 \%$ of the mass of the water molecule is in oxygen, most of the energy and momentum required to remove constituents of water from a gravity well of a celestial body goes to oxygen. It is debated how much oxygen and other heavy elements the solar wind has eroded from Mars during the age of the solar system.

The solar wind drives the erosion of ionized volatiles from globally unmagnetized Mars and Venus through their atmospheres via several channels (Lundin, 2011). The largest observed structure of the ion escape from Mars was recently termed as the heavy ion plume (Brain et al., 2010). The plume forms when the solar wind convection electric field accelerates heavy ions of atmospheric origin from ionospheric altitudes to several planetary radii distances. These planetary pickup ions follow cycloid trajectories with the Larmor radii large or comparable to the size of an induced magnetosphere in the magnetized solar wind plasma flow.

The Mars-induced magnetosphere and ion escape are hemispherically asymmetric as seen in situ observations, for example, on the Mars Express (MEX) mission (Barabash et al., 2007; Fedorov et al., 2006), and on the Mars Atmosphere and Volatile EvolutioN (MAVEN or shortly MVN) mission (Dong et al., 2015; Jakosky et al., 2015) as well as in global simulations of the Mars-solar wind interaction (Brecht, 1990; Kallio et al., 2010; Ledvina et al., 2017; Ma et al., 2015; Modolo et al., 2016; Najib et al., 2011). Typically, the near space around Mars is divided in four quadrants or hemispheres to analyze asymmetries of the ion escape (Brain et al., 2015; Nilsson et al., 2011). The plume is located in the hemisphere where the solar wind convection electric field points away from the planet, termed the $+\mathrm{E}_{\mathrm{sw}}$ hemisphere, which dominates the ion escape rate compared to the opposite $\left(-E_{s w}\right)$ hemisphere. The orientation of the plume perpendicular to the Mars-Sun axis is along the solar wind 
convection electric field. The distance heavy ions reach along the electric field is determined by their Larmor radii and, thus, by the magnitude of the interplanetary magnetic field (IMF) (Curry, Luhmann, Ma, Dong, et al., 2015; Jarvinen et al., 2016). The longer the distance, the higher the bulk energy over a Larmor period (Jarvinen \& Kallio, 2014). Further, the heavy ion plume merges continuously to the tailward escape channels when moving from the Mars magnetosheath toward the wake (Dong et al., 2015). We refer to the wake or the tail as the general region inside the induced magnetosphere boundary where no solar wind ions are observed. In addition to the induced magnetosphere boundary several boundaries have been defined between the wake and the magnetosheath, which is the region where the shocked solar wind flows downstream of the bow shock (BS). These include the magnetic pileup boundary, the ion composition boundary, the protonopause, the planetopause, and the solar wind dropout boundary (see, e.g., Barabash et al., 2007; Kallio, 2001; Matsunaga et al., 2017, and references therein).

In this work we analyze the formation of the Mars heavy ion plume as seen by multispacecraft observations and a self-consistent global hybrid simulation. In the analysis we concentrate on energization of atomic and molecular oxygen ions. We choose for analysis spacecraft orbits on the MEX and MAVEN missions with periapses $2 \mathrm{~h}$ apart from each other displaying a detection of the large-scale heavy ion plume. In the analysis we concentrate on comparing ion spectral and magnetic field observations between the spacecraft in situ instrument measurements and a virtual spacecraft in the global hybrid model. Further, we use the model to analyze finite Larmor radius (FLR) effects of planetary oxygen ions. We estimate the kinetic energies of the planetary ions as a function of distance of flight and time of flight after photoionization from the neutral exosphere or emission from the ionosphere toward the induced magnetosphere.

The following coordinate systems are used in this work. Spacecraft observations are presented in the MarsSolar Orbital coordinates, where the $x$ axis points toward the Sun from the center of the planet, the $y$ axis is antiparallel to the orbital velocity vector of Mars around the Sun, and the $z$ axis completes the right-handed coordinate system. In the model the coordinate system is similar to the Mars-Solar Orbital system with the exception that the $x$ axis points toward the solar wind flow instead of the Sun. That is, the aberration of the incident solar wind velocity vector of few degrees in the rest frame of Mars caused by the planetary orbital motion is neglected in the model. The radius of Mars $\left(R_{\mathrm{M}}=3,390 \mathrm{~km}\right)$ is used as the unit of length in the figures.

The article is organized as follows. In the next section we briefly describe the used in situ ion spectrometer and magnetic field observations on the MEX and MAVEN missions. Then we present the RHybrid global hybrid simulation code. In the results section we present results from our numerical modeling experiment and compare them to the observations. At the end we discuss and summarize our findings.

\section{Spacecraft Observations}

An orbit from MAVEN and an orbit from MEX on 25 January 2015 were chosen for analysis as ion spectrometers on both spacecraft showed a clear detection of the heavy ion plume on that day (Figures $3 \mathrm{c}$ and $3 \mathrm{~g}$ ). The spacecraft orbit geometries are shown in Figure 1. MEX is close to the noon-midnight orbit configuration, whereas MAVEN's trajectory is between the terminator and noon-midnight planes. Observational data from several instruments on the MAVEN and MEX mission are analyzed here.

The Analyzer of Space Plasma and Energetic Atoms (ASPERA-3) particle package on MEX (Mars orbit insertion in 2003) includes the lon Mass Analyzer (IMA) mass-resolving ion spectrometer (Barabash \& Lundin, 2006). ASPERA-3/IMA measures ions in the energy range from $10 \mathrm{eV} / \mathrm{e}$ to $36 \mathrm{keV} / e$, where $e$ is the positive elementary charge, with the nominal time resolution of $196 \mathrm{~s}$. Suprathermal and Thermal lon Composition (STATIC) (McFadden et al., 2015) and Solar Wind Ion Analyzer (SWIA) (Halekas et al., 2015) ion spectrometers on MAVEN (Mars orbit insertion in 2014) have both intrinsic cadence of $4 \mathrm{~s}$. STATIC is a mass-resolving instrument with the energy range from $0.1 \mathrm{eV} / \mathrm{e}$ to $30 \mathrm{keV} / \mathrm{e}$, whereas SWIA measures all ions without mass separation capability in the energy range from $5 \mathrm{eV} / \mathrm{e}$ to $25 \mathrm{keV} / \mathrm{e}$. Thus, MAVEN's modern ion spectrometers are able to measure ions at lower energies and higher time resolution than the ion spectrometer on MEX. MAVEN's magnetometer (MAG) uses triaxial fluxgate sensors operating at an intrinsic sampling rate of 32 vector measurements per second, while MEX does not have a magnetometer.

Figure 2 displays the magnetic field observed by MAVEN's magnetometer (Connerney et al., 2015). MVN/SWIA, MVN/STATIC and MEX/ASPERA-3/IMA observations are plotted in Figure 3. Structures of the Martian-induced magnetosphere are evident in magnetic and particle observations and they are described in the Results section. 

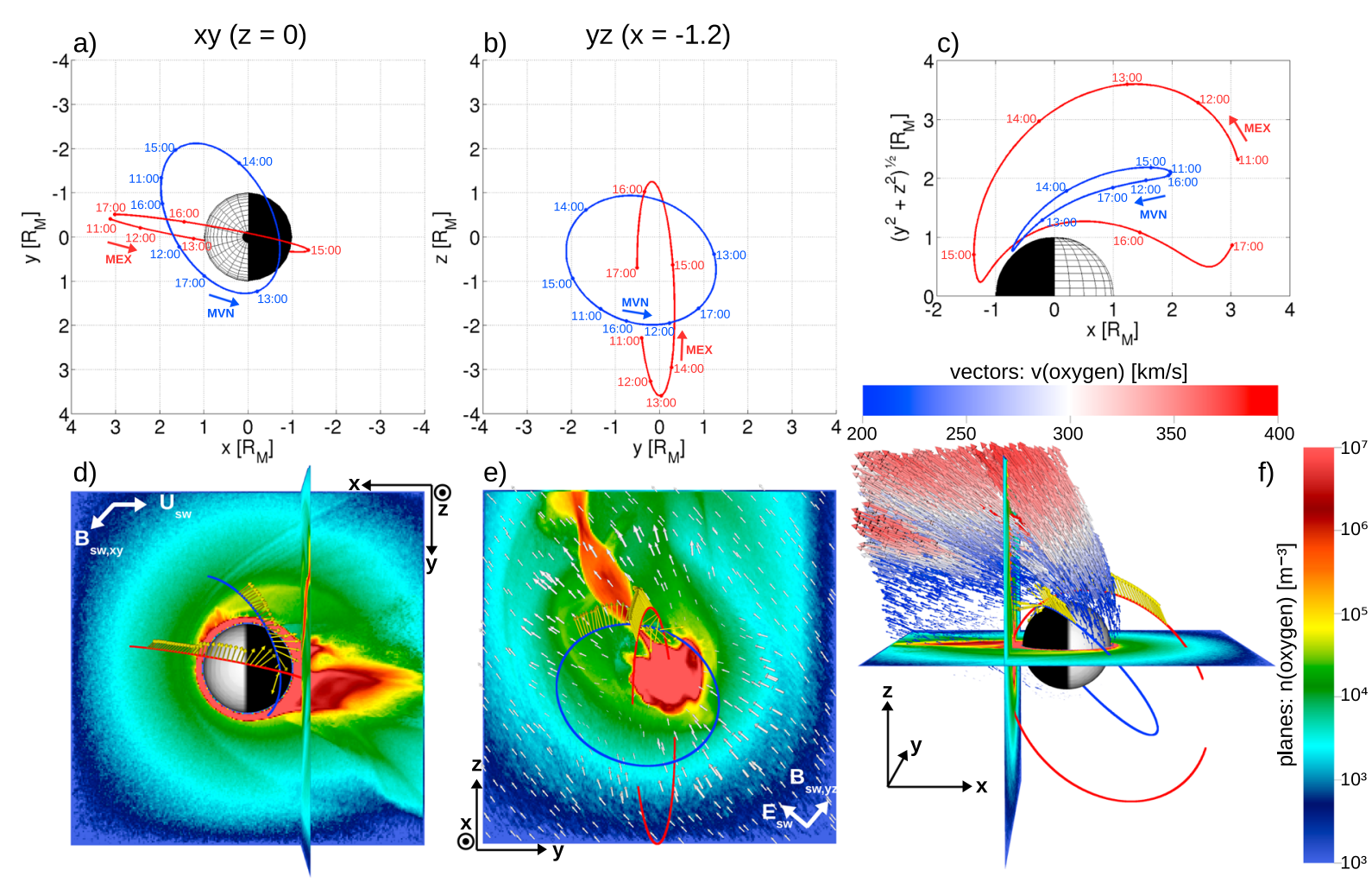

Figure 1. (a-c) MEX (red line) and MAVEN(blue line) orbits on 25 January 2015 analyzed in this study. (d-f) Escaping oxygen ions (ionospheric $\mathrm{O}^{+}$and $\mathrm{O}_{2}^{+}$ions and exospheric $\mathrm{O}^{+}$photoions) from Mars in the simulation Run 2. Colored surfaces give Martian oxygen ion density at $z=0$ and $x=-1.2 R_{\mathrm{M}}$ planes. (d-f) Yellow arrows on the orbit curves are oxygen ion velocity unit vectors in the simulation in the region where the ion plume was observed by the spacecraft. (e) White arrows show the electric field at $x=-1.2 R_{\mathrm{M}}$ plane. (f) Colored arrows display oxygen ion velocity vectors in the regions of highest oxygen ion flux $\left(n v>10^{10} \mathrm{~m}^{-2} \mathrm{~s}^{-1}\right)$. ( $\left(\mathrm{d}\right.$ and e) $\mathrm{U}_{\mathrm{sw}}, \mathrm{B}_{\mathrm{sw}}$, and $\mathrm{E}_{\mathrm{sw}}$ arrows display the orientation of the undisturbed upstream solar wind flow, magnetic field, and electric field, respectively.

\section{Global Hybrid Code: RHybrid}

We use a highly parallel $\mathrm{C}++$ global hybrid simulation platform for planetary plasma interactions named RHybrid, which was recently developed at the Finnish Meteorological Institute (FMI) (Jarvinen \& Sandroos, 2013). In the code ions are modeled as grid cell sized and shaped macroscopic particle clouds (macroparticles or macroions) moving under the Lorentz force. Trilinear macroparticle weighting is applied when ion moments are estimated in the Cartesian simulation mesh of cubic grid cells. Grid cells and particles are distributed to computing cores via the Message Passing Interface (MPI) based on the FMI's ParGrid and Corsair high-performance computing simulation libraries (Hietala et al., 2012; Sandroos, 2013). Computational load is dynamically balanced between the MPI processing elements during a simulation run by the Zoltan library using the Recursive Coordinate Bisection algorithm (Boman et al., 2012). In this work the RHybrid code was run on 720 computing cores of the FMI's Cray XC30 system for about 16k core hours per simulation run.

In the simulation runs analyzed here the magnetic field $(\vec{B})$ is stored on grid cell faces and the electric field $(\vec{E})$ is estimated on grid cell nodes and edges. This Yee type staggered grid guarantees divergence-free propagation of magnetic field according to Faraday's law (Dai \& Woodward, 1998). Electrons are treated as a massless and pressureless fluid, which provides quasi-neutrality of the plasma. Thus, the electron dynamics are governed by the equations $q_{\mathrm{e}} n_{\mathrm{e}} \vec{U}_{\mathrm{e}}=\vec{J}-\sum_{i} q_{i} n_{i} \vec{V}_{i}$ and $q_{\mathrm{e}} n_{\mathrm{e}}=\sum_{i} q_{i} n_{i}$, where $q_{\mathrm{e}}, n_{\mathrm{e}}$, and $\vec{U}_{\mathrm{e}}$ are the electron charge, number density, and velocity, $q_{i}, n_{i}$, and $\vec{V}_{i}$ are the ion charge, number density, and velocity with the summation $i$ over all macroions in a grid cell, and $\vec{J}$ is the total current density defined by Ampere's law. Thus, the summed electron $\left(q_{\mathrm{e}} n_{\mathrm{e}} \vec{U}_{\mathrm{e}}\right)$ and ion $\left(\sum_{i} q_{i} n_{i} \vec{V}_{i}\right)$ current densities in a grid cell are equal to the total current density, while the total charge density in a grid cell remains zero.

The magnetic field is propagated by Faraday's law $\partial \vec{B} / \partial t=-\nabla \times \vec{E}$, where the Ohm's law reads $\vec{E}=-\overrightarrow{U_{\mathrm{e}}} \times \vec{B}+\eta \vec{J}$ and $\eta$ is a given explicit resistivity profile. The resistivity is used to add diffusion in the propagation of the 


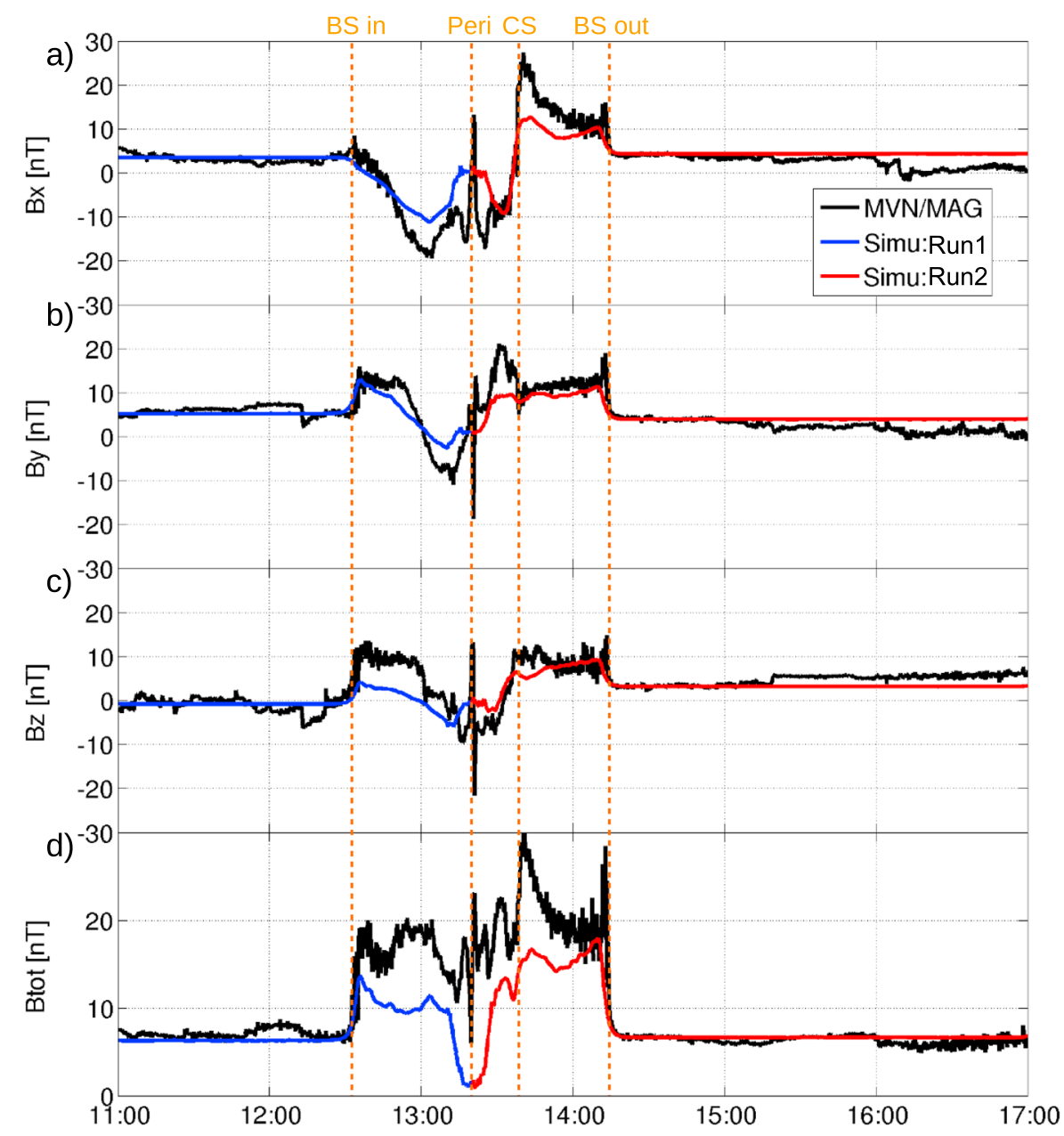

Figure 2. MAVEN's magnetometer observations (black line) compared to the simulation Run 1 with inbound upstream conditions (blue line) and Run 2 with outbound conditions (red line). Orange vertical lines indicate the crossing of the inbound bow shock (BS in), the crossing of the outbound bow shock (BS out), the periapsis of MAVEN orbit (Peri) and the crossing of the central tail current sheet (CS).

magnetic field to increase numerical stability, while the resistive electric field term is not included explicitly in the Lorentz force. The magnetic diffusion time scale determined by the induction equation is $\tau_{d}=\mu_{0} L_{B}^{2} / \eta$, where $\mu_{0}$ is the permeability of free space and $L_{B}$ is the scale length of the magnetic field (e.g., Koskinen, 2011). The value of $\eta$ above the inner boundary is set as $\eta=0.02 \mu_{0} \Delta x^{2} / \Delta t$, where $\Delta x$ is a grid cell side length and $\Delta t$ is the time step (see Table 1). By setting $L_{B}=\Delta x$, which is the smallest possible scale length of the magnetic field in a simulation, the lower limit of the magnetic diffusion time comes $\tau_{d}=\Delta t / 0.02=50 \Delta t$. That is, $\eta$ allows the diffusion of the magnetic field only on time scales much longer than the simulation time step.

In the analyzed runs the ionospheric obstacle to the solar wind flow is modeled as a superconducing sphere. This is implemented by setting the resistivity as zero inside the inner boundary. Thus, $\tau_{d}$ becomes infinite inside the inner boundary and the magnetic field does not diffuse through the obstacle. Further, ions impacting the inner boundary are absorbed and removed from the simulation and the electron velocity is set as zero inside the inner boundary.

The simulation domain is surrounded by a layer of passive cells called ghost cells, where boundary conditions are enforced. The solar wind is implemented at the front wall by injecting moving Maxwellian populations of $\mathrm{H}^{+}$and $\mathrm{He}^{++}$ions. The perpendicular component of the IMF to the plasma flow is held constant in the ghost cells of the front wall and, thus, convects in the domain with the solar wind. The flow-aligned component of the IMF is implemented as an initial magnetic field derived from potential flow, which has zero radial component 

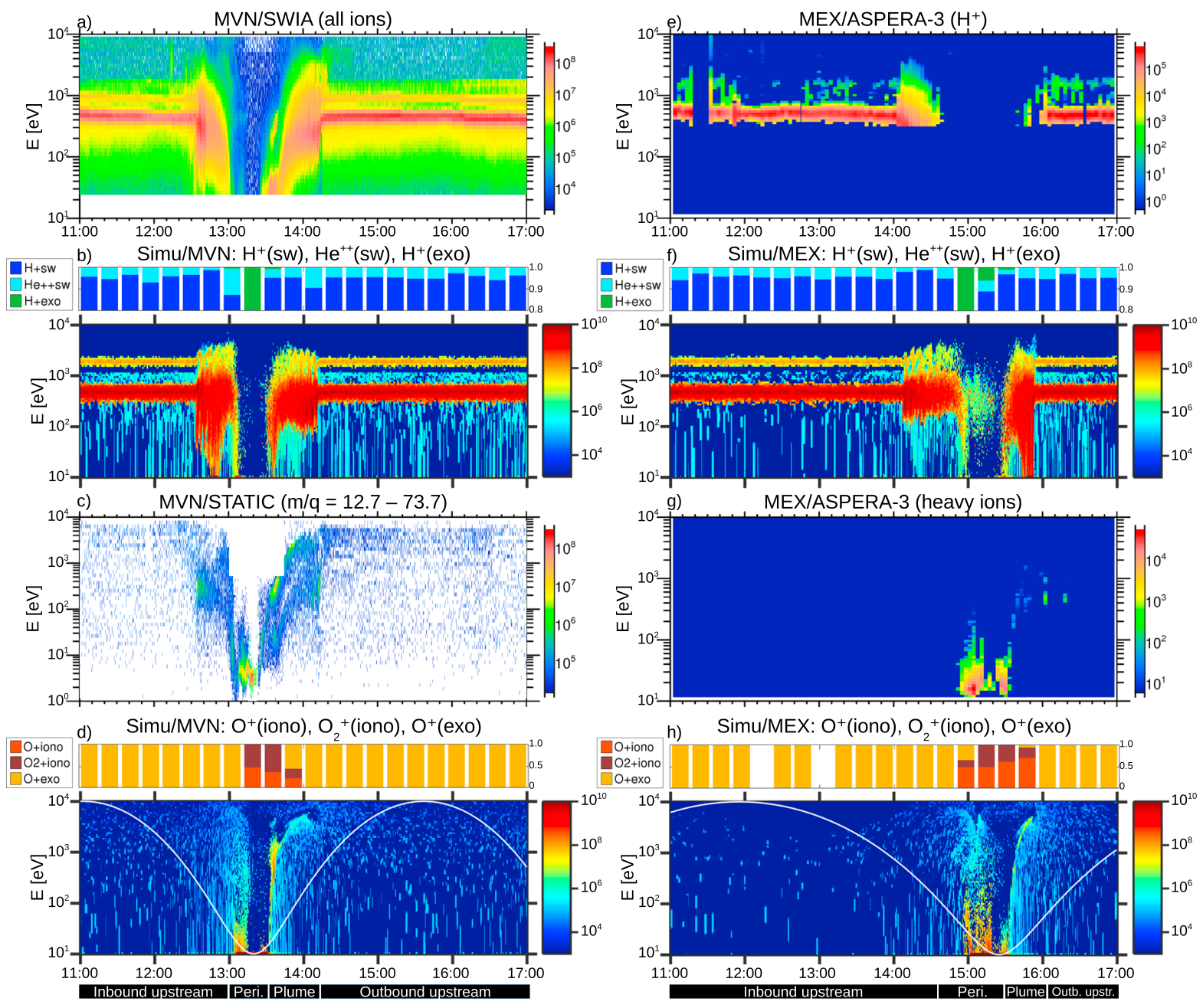

Figure 3. Ion time-energy spectrograms observed by MAVEN (a-d) and MEX (e-h) compared to the simulation Run 2. Observations show all MAVEN/SWIA ions, MAVEN/STATIC heavy ions, MEX/ASPERA-3/IMA protons, and MEX/ASPERA-3/IMA heavy ions. The model spectra show omnidirectional differential ion flux gathered along the spacecraft trajectories with the coloring determined as $\sum_{i} w_{i} v_{i} /(4 \pi \Delta V \Delta E)\left[\# /\left(\mathrm{s} \mathrm{m} \mathrm{m}^{2} \mathrm{eV}\right.\right.$ sr)], where the summation $i$ is over macroions in a spatial cell ( $\Delta V$ is the cell volume) and energy range $[E, E+\Delta E], w_{i}$ is the statistical weight of a macroion, $v_{i}$ is the speed of a macroion, and the full solid angle is used to normalize the flux. The MVN/SWIA and MVN/STATIC plots are in the units of differential ion energy flux [eV/(s $\mathrm{cm}^{2} \mathrm{eV} \mathrm{sr)],} \mathrm{whereas} \mathrm{MEX/ASPERA-3/IMA}$ plots are in the units of differential ion flux [\#/( $\left.\left(\mathrm{cm}^{2} \mathrm{eV} \mathrm{sr}\right)\right]$. Above the model spectra relative ion concentrations are shown for the solar wind protons and alphas $\left(\mathrm{H}^{+}(\mathrm{sw})\right.$ and $\left.\mathrm{He}^{++}(\mathrm{sw})\right)$, exospheric photoionized protons $\left(\mathrm{H}^{+}(\mathrm{exo})\right)$, atomic and molecular oxygen ions emitted from the ionosphere $\left(\mathrm{O}^{+}(\right.$iono $)$and $\mathrm{O}_{2}^{+}($iono $\left.)\right)$, and exospheric photoionized atomic oxygen ions $\left(\mathrm{O}^{+}(\mathrm{exo})\right)$. White curves in panels $(\mathrm{d})$ and $(\mathrm{h})$ show the evolution of the spacecraft altitude as a function of time for context. The linear altitude curves (the linear scale not shown) are fitted in the panels in such a way that the orbit apoapses coincide with the maximum value of the vertical axis and the orbit periapsis coincide with the minimum value of the vertical axis (see the orbits in Figure 1). At the bottom are denoted the inbound upstream, periapsis, plume, and outbound upstream intervals.

at the inner boundary and is homogeneous at infinity (Kallio et al., 2006; Shimazu, 1999). This field corresponds to a laminar flow around a sphere.

Planetary ions are injected in the simulation from the inner boundary (Mars ionospheric emission) and via photoionization of the Mars exosphere. Both atomic $\left(\mathrm{O}^{+}\right)$and molecular $\left(\mathrm{O}_{2}^{+}\right)$oxygen ions are emitted from the ionosphere, and $\mathrm{H}^{+}$and $\mathrm{O}^{+}$photoions are created from the exosphere. The ionospheric emission has a maximum flux at noon and $\cos (\mathrm{SZA})$, where SZA is the solar zenith angle, dependence toward terminator where the flux reaches $10 \%$ of the noon value and is constant for the nightside. The exospheric photoionization rate is constant above the inner boundary except in the shadow of the planet where no photoions are created. Oxygen and hydrogen neutral corona profiles are taken from the "Run C (solar maximum with exosphere)" input definitions of the "Intercomparison of Global Models and Measurements of the Martian Plasma Environment" 
Table 1

Simulation Configuration and Upstream Solar Wind (SW) and Interplanetary Magnetic Field (IMF) Conditions

Parameter - Value

Box size $(x \times y \times z)\left[R_{M}\right]-(-4 \ldots 4) \times(-4 \ldots 4) \times(-4 \ldots 4)$

Number of grid cells $\left(n_{x} \times n_{y} \times n_{z}\right)-240 \times 240 \times 240$

Grid cell size $\left(\Delta x^{3}\right)-\left(R_{M} / 30\right)^{3}=(113 \mathrm{~km})^{3}$

Average number of macroparticles per cell - 85

Time step $(\Delta t)-7.5 \mathrm{~ms}$

Solution snapshot time - $600 \mathrm{~s}$

SW velocity $\left[V_{x}, V_{y}, V_{z}\right]-[-300,0,0] \mathrm{km} \mathrm{s}^{-1}$

SW ion temperature $-60,000 \mathrm{~K}$

$\mathrm{O}^{+}$ionospheric emission rate $-4.2 \times 10^{25} \mathrm{~s}^{-1}$

$\mathrm{O}_{2}^{+}$ionospheric emission rate $-6.0 \times 10^{25} \mathrm{~s}^{-1}$

$\mathrm{O}^{+}$exospheric production rate $-1.57 \times 10^{24} \mathrm{~s}^{-1}$

$\mathrm{H}^{+}$exospheric production rate $-3.64 \times 10^{24} \mathrm{~s}^{-1}$

lonospheric emis. radius $-R_{\mathrm{V}}+400 \mathrm{~km}$

Zero resistivity (obstacle) radius $-R_{\mathrm{V}}+300 \mathrm{~km}$

Particle absorption radius $-R_{\mathrm{V}}+200 \mathrm{~km}$

Run 1 (MAVEN ib)

IMF $\left[B_{x}, B_{y}, B_{z}\right]-[3.5,5.3,-0.8] \mathrm{nT}$

IMF magnitude - 6.4 nT

IMF cone angle $-57^{\circ}$

IMF clock angle $-98^{\circ}$

SW ion density $\left[\mathrm{cm}^{-3}\right]-n\left(\mathrm{H}^{+}\right)=15, n\left(\mathrm{He}^{++}\right)=0.75$

Run 2 (MAVEN ob and MEX ib)

IMF $\left[B_{x}, B_{y}, B_{z}\right]-[4.3,4.0,3.3] \mathrm{nT}$

IMF magnitude - $6.7 \mathrm{nT}$

IMF cone angle $-51^{\circ}$

IMF clock angle $-50^{\circ}$

SW ion density $\left[\mathrm{cm}^{-3}\right]-n\left(\mathrm{H}^{+}\right)=20, n\left(\mathrm{He}^{++}\right)=1.0$

Note. Run 1 uses the conditions determined at the MAVEN inbound (ib) bow shock crossing and Run 2 uses the conditions determined at the MAVEN outbound (ob) and MEX inbound bow shock crossing. The cone angle is defined as $\arctan \left(\sqrt{B_{y}^{2}+B_{z}^{2}}, B_{x}\right)$, and the clock angle is defined as $\arctan \left(B_{y}, B_{z}\right)$.
International Space Science Insitute (ISSI) team's second meeting (Modolo et al., 2016). The total ionospheric emission rates were chosen so that they best fit the spacecraft orbits analyzed here (see, e.g., Jarvinen et al., 2009). This meant that the ionospheric emission rates were increased by a factor of 3 for this study compared to the ionospheric emission in an earlier run where solar minimum exospheric photoionization conditions from ISSI team's first meeting were used (Kallio et al., 2010).

Kallio and Janhunen (2003) describe details of the used numerical algorithm with the following exceptions. In RHybrid the electron velocity is determined directly at grid cell nodes, which means no cell volume average to node interpolation is needed. The electric current density is evaluated at the edges of the grid cell faces instead of the grid cell nodes (Pohjola \& Kallio, 2010). Further, no artificial macroparticle splitting and joining nor adaptive mesh refinements are used in RHybrid. The number of particles and grid cells can be much higher in parallelized than sequential simulation codes.

Table 1 lists the most important simulation parameters and upstream conditions of the RHybrid simulation runs analyzed in this work. Run 1 describes the MAVEN inbound upstream conditions, and Run 2 describes the MAVEN outbound and MEX inbound upstream conditions for the analyzed orbit case. The simulation was run until the Martian-induced magnetosphere and plasma environment were formed and the simulation had reached near stationary development after initial transients.

\section{Results}

Figures $1 d-1 f$ display the number density and bulk velocity of oxygen ions in the simulation Run 2. Photoions originating from the exosphere are seen away from near vicinity of the planet as a spherically symmetric distribution (light blue and green), while in the nightside and close to the planet the dense plasma sheet and the heavy ion plume of ionospheric origin are evident (red). The plasma sheet and the plume are oriented along the electric field shown as white arrows in Figure 1e. Regions of highest oxygen flux (blue-red velocity vectors in Figure 1f) include mostly the plume, the plasma sheet, and the magnetosheath on the $+\mathrm{E}_{\mathrm{sw}}$ hemisphere. The velocity increases in concert with increasing distance along the electric field both in the dayside and in the nightside outside of the wake. The plume sampled by MAVEN (indicated by yellow arrows on blue spacecraft trajectory) crosses some of these high flux regions in the model, whereas the plume sampled by MEX (indicated by yellow arrows on red spacecraft trajectory) is mostly not in the high flux regions in the model.

\subsection{Magnetic Field}

Figure 2 compares the magnetic field observed by MAVEN with both simulation runs. Run 1 (inbound upstream conditions) is plotted until periapsis and Run 2 (outbound upstream conditions) from periapsis. Crossing of the quasi-perpendicular region of the BS is seen as abrupt jumps in the magnetic field at 12:30 (BS in) and 14:15 (BS out) both in the observations and in the model. The periapsis occurs at 13:20 (Peri), and the field has a local minimum and a temporary sign change in all components when the spacecraft is in the ionosphere. In the model the magnitude of the magnetic field decreases already for a few minutes before and after the periapsis when the orbit is inside the inner boundary of the model (13:12-13:27). The central tail current sheet is crossed at 13:40 when the field magnitude has a local minimum and the $B_{x}$ and $B_{z}$ components change sign both in the observations and in the model.

\subsection{Ion Spectra}

Figure 3 compares observed and modeled ion time-energy spectrograms of heavy and light ions along trajectories of both spacecraft. In Figures $3 \mathrm{a}$ and $3 \mathrm{e}$, and $3 \mathrm{~b}$ and $3 \mathrm{f}$ the upstream beams of the solar wind $\mathrm{H}^{+}$and $\mathrm{He}^{++}$ions and the planetary environment with the shocked solar wind plasma are evident. Heating of the 

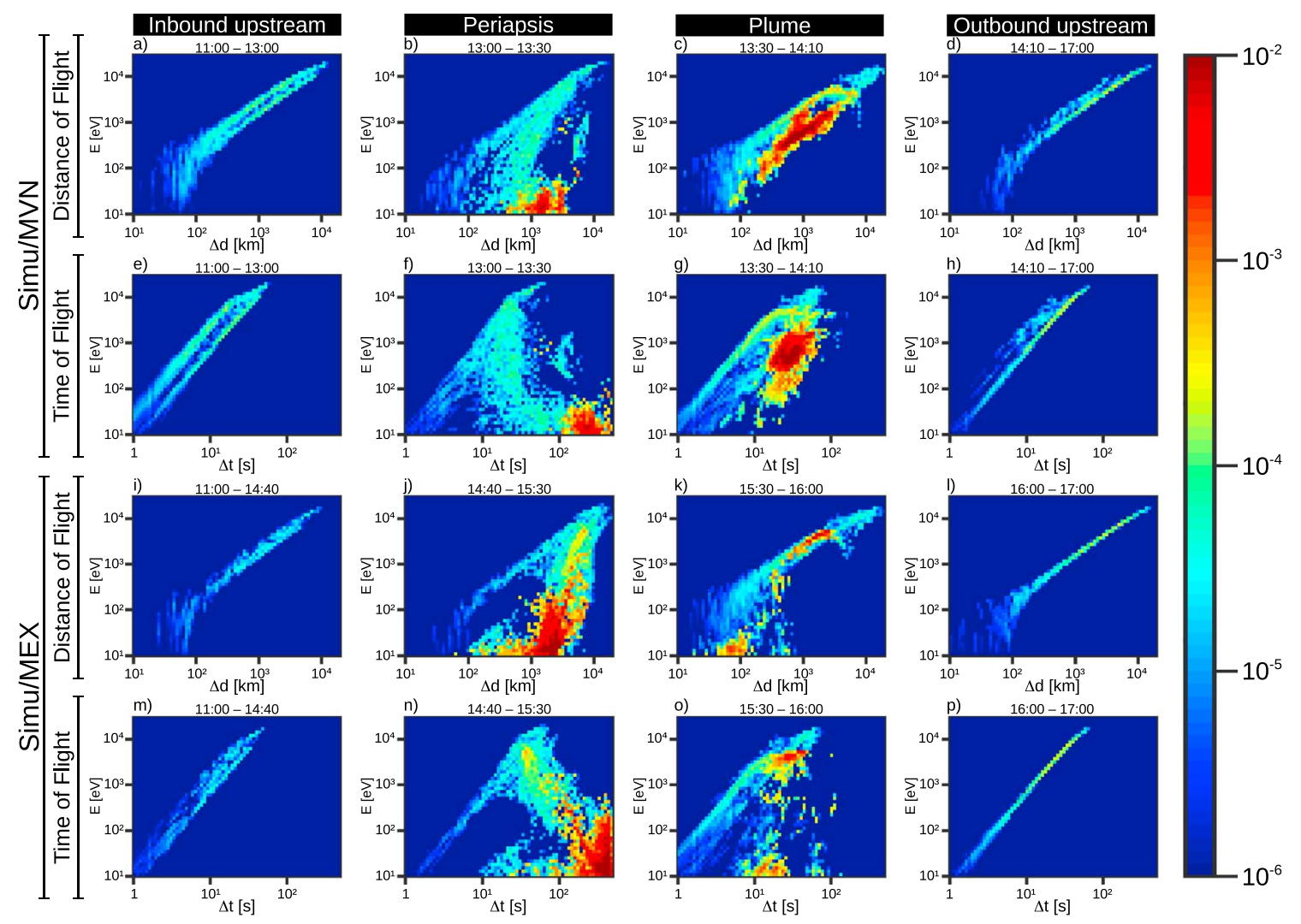

Figure 4. Probability density maps of the oxygen ion energy $(E)$ as a function of the distance of flight $(\Delta d=$ DoF) and the time of flight $(\Delta t=$ ToF) along the spacecraft trajectories in the simulation Run 2. Ion counts at four time intervals (inbound upstream, periapsis, plume, and outbound upstream) from panels (d) and (h) of Figure 3 are included in these maps. $\Delta d$ is the distance between the observation point and the ionization point. $\Delta t$ is the duration from the ionization time to the observation time. The color scale is determined as $\sum_{i} w_{i} / w_{\text {all }}$, where the summation $i$ is over ions in spatial cells along the spacecraft orbit in the time interval and in phase space cells $(\Delta d, \Delta E)$ and $(\Delta t, \Delta E), w_{i}$ is the weight of an ion, and $w_{\text {all }}$ is the sum of all ion weights in the spectra.

upstream solar wind ion beams occurs at the BS. Near the periapsis the solar wind dropout boundaries occur at 13:00 and 13:30 UT along the MAVEN trajectory and at 14:30 and 15:40 along the MEX trajectory both in the model and in the observations. The wake region is mostly void of light ions. Exospheric $\mathrm{H}^{+}$photoions are seen in the model spectra as light blue scattered counts. Further, the modeled light ion composition is dominated by the solar wind $\mathrm{H}^{+}$with a minor $\mathrm{He}^{++}$component except near the periapsis where the composition is dominated by sporadic exospheric $\mathrm{H}^{+}$ion counts in the wake (green in Figures $3 \mathrm{~b}$ and $3 \mathrm{f}$ ).

In Figures $3 c$ and $3 g$, and $3 d$ and $3 \mathrm{~h}$ heavy ions are analyzed. Lowest energies ( $\lesssim 10 \mathrm{eV}$ ) occur in and near the ionosphere (periapsis) in the observations and in the model. Exospheric $\mathrm{O}^{+}$photoions are seen in the model as light blue scattered counts through out the spectra. After the periapsis crossing the heavy ion plume is seen as increasing energies from $10 \mathrm{eV}$ up to several keVs (MAVEN: 13:20-13:50 UT and MEX: 15:30-15:50 UT). In the simulation the heavy ion composition near the periapsis and the plume is dominated by the $\mathrm{O}^{+}$and $\mathrm{O}_{2}^{+}$ions of ionospheric origin. The exospheric $\mathrm{O}^{+}$photoions dominate the composition elsewhere.

\subsection{Distance and Time of Flight Analysis}

Figure 4 analyses the oxygen ion energization in the simulation Run 2 along the spacecraft trajectories using two-dimensional histograms of distance of flight (DoF) and time of flight (ToF) versus particle energy. The orbits of both spacecraft are divided in four parts for detailed analysis of the ion dynamics in different plasma regions around Mars as shown in Figure 3 (columns from left): inbound upstream (Figures 4a, 4e, 4i, and $4 \mathrm{~m}$ ), periapsis/ionosphere (Figures $4 \mathrm{~b}, 4 \mathrm{f}, 4 \mathrm{j}$, and $4 \mathrm{n}$ ), plume (Figures $4 \mathrm{c}, 4 \mathrm{~g}, 4 \mathrm{k}$, and 4o), and outbound upstream (Figures 4d, 4h, 4l, and 4p).

The DoF and ToF energy histograms were created as follows. Macroparticles in every grid cell touching the spacecraft orbits were recorded until $25 \times 10^{6}$ macroions were reached per orbit. This took about 147 time steps (1.1 s) with about 500 cells per orbit within the simulation domain. Each macroparticle carried the 
following extra information for the analysis: simulation time, particle coordinates, and velocity at its injection (or ionization) in the simulation. Together with simulation time, particle coordinates and velocity at its observation (or collection) and the statistical weight of the particle the histograms were constructed. The DoF $(\Delta d)$ was determined as the straight distance between the observation point and the injection point of a particle. Similarly, the ToF $(\Delta t)$ was determined as the duration between the observation time and the injection time of a particle. The particle energy $(E)$ was determined from its velocity at the collection. Injection velocities were generally very low compared to the velocites at observation, which means that the particle energy in Figure 4 is almost the same as the energy gained by the particle since its injection in the simulation $\left(E_{\text {observation }} \approx \Delta E=E_{\text {observation }}-E_{\text {injection }}\right)$.

Figure 4 reveals several details about oxygen ion energization in the simulation. The ion energy as a function of the distance of flight in Figures $4 a-4 d$ (MAVEN) and Figures $4 i-4 l$ (MEX) show that the more distance the particle has to its ionization point when collected in a grid cell on the spacecraft orbit, the higher the particle energy in general. The same is true for the time of flight (Figures $4 \mathrm{e}-4 \mathrm{~h} / \mathrm{MAVEN}$ and Figures $4 \mathrm{~m}-4 \mathrm{p} / \mathrm{MEX}$ ): the more time the particle has spent in the simulation when collected, the higher the energy in general. The correlation is almost approximately linear in log-log scale especially in the upstream solar wind regions (Figures $4 a-4 e, 4 i$, and $4 m$ and Figures $4 d-4 h, 4 l$, and $4 p$ ). In the upstream region the oxygen ion composition is purely exospheric $\mathrm{O}^{+}$as seen in Figures 3 and $3 \mathrm{~h}$.

Also more complicated dependencies between the DoF and the ToF and the ion energy occur in the simulation. The histograms display nonlinearity in the log-log scale mostly near the planet for both spacecraft orbits. A strong source of oxygen ions emitted from the ionosphere is seen as red regimes along both orbits in the periapsis and plume columns of Figure 4, while the light blue, green, and yellow regimes are mostly exospheric oxygen photoions (separate figures for ionospheric and exospheric populations not shown). The oxygen ions emitted from the ionosphere in the periapsis column of both spacecraft are at lower energies than in the plume column as expected. In the MAVEN periapsis column the red ionospheric population has energies of about 10-50 eV and DoF of 1,000-2,000 km (Figure 4b) and ToF of 100-300 s (Figure 4f). In the MEX periapsis column the red ionospheric population has a broader spread in energy of $\approx 10-200 \mathrm{eV}$ likely due the higher periapsis altitude of MEX than MAVEN allowing higher energies. The ionospheric population in the MEX periapsis has DoF of 1,000-10,000 km (Figure 4j) and ToF of 100-400 s (Figure 4n).

In the plume column of both spacecraft the red ionospheric population has moved to higher energies and displays more linear or monotonously increasing features as a function of DoF and ToF than in the periapsis column. Along the MEX orbit the ionospheric plume overlaps with the exospheric photoions above $1 \mathrm{keV}$ (Figures $4 \mathrm{k}$ and 40 ). Along the MAVEN orbit the ionospheric plume is seen at somewhat lower energies than the exospheric photoions (Figures $4 \mathrm{c}$ and Figure $4 \mathrm{~g}$ ). Low energy ionospheric oxygen at $10 \mathrm{eV}$ and DoF $=100$ $\mathrm{km}$ and $\mathrm{ToF}=10-20 \mathrm{~s}$ are seen in the MEX plume column (Figures $4 \mathrm{k}$ and 40 ). These can be associated with the ionospheric emission from the low-altitude night side wake region where the acceleration of heavy ions is less efficient than higher up and away from the wake.

\section{Discussion}

MAVEN and MEX observations at Mars on 25 January 2015 show the heavy ion plume signature in the $+\mathrm{E}_{s w}$ hemisphere. In the model the ionospheric heavy ion plume is also evident along the trajectories of both spacecraft. MEX sampled the plume near the noon-midnight orbit configuration, whereas MAVEN sampled the plume along the orbit between the terminator plane and the noon-midnight plane. The plume observations were about $2 \mathrm{~h}$ apart from each other between MAVEN and MEX. This indicates that the plume is a stable structure over temporal scale of at least few hours provided that the orientation of the large-scale solar wind convection electric field remains stable. This is in agreement with single spacecraft studies and models (Brain et al., 2010; Curry, Luhmann, Ma, Liemohn, et al., 2015; Dubinin et al., 2008; Dong et al., 2015; Jarvinen et al., 2016).

In the simulation the energization of oxygen ions starts at energies of $\lesssim 10 \mathrm{eV}$ and reaches several keVs in the Mars-induced magnetosphere along the spacecraft trajectories. In the upstream solar wind region, where the pure ion pickup is the large-scale acceleration process, the correlation of distance and time of flight (DoF/ToF) with the oxygen ion energy is linear on a log-log scale according to the model. The linearity goes up to $\sim 20,000 \mathrm{~km}$ and $\sim 30 \mathrm{~s}$ when oxygen ions have reached the energy of $10 \mathrm{keV}$ along these spacecraft trajectories. Also, other parts of the orbits display monotonously increasing structures in the oxygen energies 


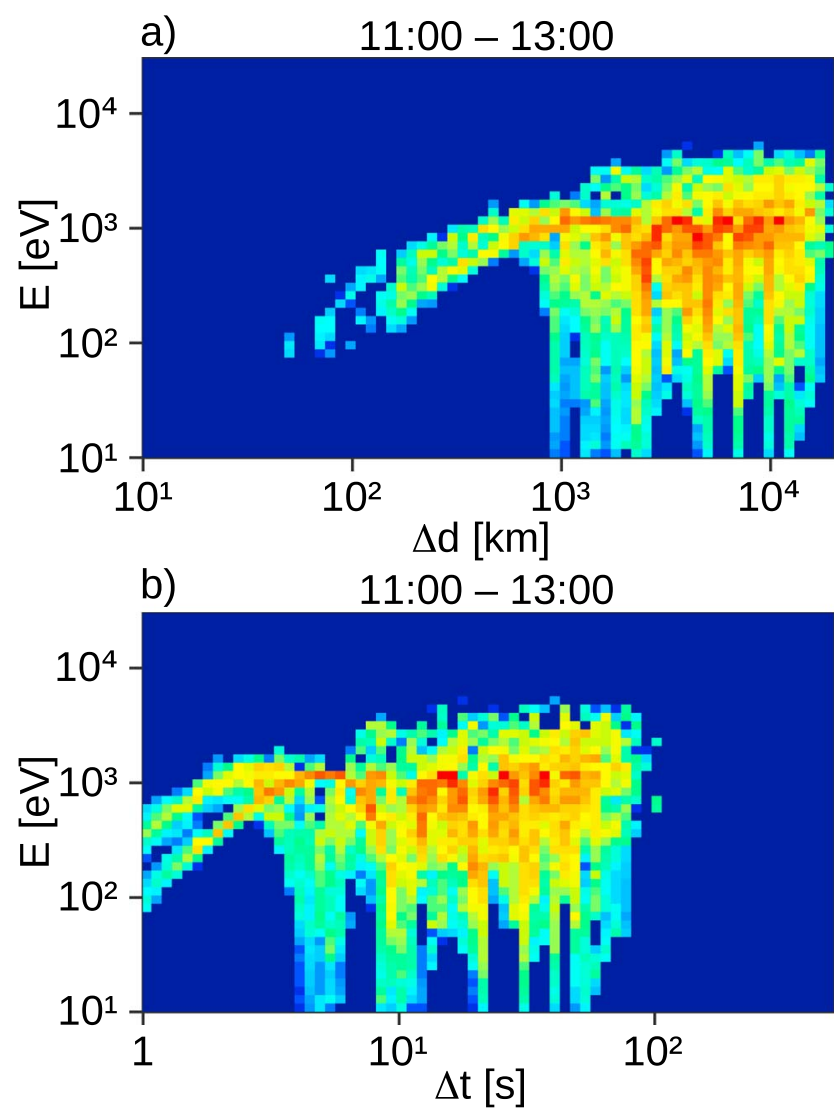

Figure 5. Probability density maps of the exospheric photoionized hydrogen ion energy $(E)$ as a function of the distance of flight $(\Delta d)$ and the time of flight $(\Delta t)$ along the MAVEN trajectories in the simulation Run 2. Ion counts of $\mathrm{H}^{+}$(exo) in Figure $3 \mathrm{~b}$ are included in these maps. The figure is in the same format and color scale as panels (a) and (e) of Figure 4. as a function of DoF and ToF. This implies that oxygen ions are within the first half of their gyrophase after ionization from the exosphere or emission from the ionosphere. Escaping oxygen ions have not had time to get accelerated to higher gyrophases as the periapses of the spacecraft orbits analyzed here are on the $+\mathrm{E}_{\mathrm{sw}}$ hemisphere. Higher oxygen ion energies and gyrophases would be observed during orbits with the apoapsis on the $+E_{s w}$ hemisphere. All in all, oxygen ions display strong FLR behavior in their energization.

Let us consider the FLR effects and the mass-to-charge ratio of the planetary ion species in light of our DoF and ToF analysis. The Larmor period and radius of a charged particle are $\tau_{\mathrm{L}}=2 \pi m /(q B)$ and $r_{\mathrm{L}}=m V_{\perp} /(q B)$, respectively, where $m$ and $q$ are the mass and electric charge of the particle and $V_{\perp}$ is the velocity of the particle perpendicular to the magnetic field. The ratio for atomic oxygen ions is 16 times the ratio of the protons. Thus, protons should undergo 16 gyrations in the same time when atomic oxygen ions undergo one, provided that electric and magnetic field are the same and particles start at rest. Also, $\mathrm{H}^{+}$have the Larmor radius of one sixteenth times the Larmor radius of $\mathrm{O}^{+}$. The gyromotion of planetary pickup protons along the space should maybe be more pronounced than the gyromotion of oxygen, which only displays less than about the first half of the first gyrophase of oxygen pickup ions as discussed in the previous paragraph.

Figure 5 shows the DoF and ToF versus particle energy histograms of the exospheric hydrogen photoions becoming picked up by the upstream solar wind flow along the MAVEN trajectory in the inbound upstream region. These can be compared to Figures $4 a$ and $4 \mathrm{e}$ for oxygen ions. The motion over several gyroperiods can be seen for planetary protons. All proton gyrophases are evident in the plot as kinetic energies are not monotonously related to DoF nor ToF. The gyromotion of the pickup protons occurs at spatial and temporal scales small enough that gyrophases of their multiple gyrations around the magnetic field are seen along the analyzed spacecraft orbits around Mars. This can be estimated as follows.

Let us consider the pickup ion (PUI) dynamics in the single-particle picture in homogeneous electric and magnetic fields (e.g., Jarvinen \& Kallio, 2014) corresponding to upstream conditions of Run 2 (see numerical values of the parameters in Table 1). $\mathrm{An} \mathrm{O}^{+}$ion picked up from rest $\left(\mathrm{O}_{\mathrm{PUI}}^{+}\right)$by the undisturbed solar wind has a gyroradius and period of $r_{\mathrm{L}}\left(\mathrm{O}_{\mathrm{PUI}}^{+}\right)=m_{\mathrm{O}^{+}} V_{E \times B} /(e B)=5,720 \mathrm{~km}$ and $\tau_{\mathrm{L}}\left(\mathrm{O}_{\mathrm{PUI}}^{+}\right)=2 \pi m_{\mathrm{O}^{+}} /(e B)=155 \mathrm{~s}$, where $m_{\mathrm{O}^{+}}$is the oxygen mass and the drift velocity reads $V_{E \times B}=\vec{E} \times \vec{B} / B^{2}$ and $\vec{E}=-\vec{V} \times \vec{B}$. At the middle of the gyrophase the particle reaches the maximum energy of $E_{\mathrm{kin}}^{\max }\left(\mathrm{O}_{\mathrm{PUI}}^{+}\right)=4\left(0.5 \mathrm{~m}_{\mathrm{O}^{+}} V_{E \times B}^{2}\right)=18 \mathrm{keV}$ after $\tau_{\mathrm{L}}\left(\mathrm{O}_{\mathrm{PUI}}^{+}\right) / 2=78 \mathrm{~s}$ from ionization and after moving $d_{\mathrm{L}}\left(\mathrm{O}_{\mathrm{PUI}}^{+}\right) / 2=22,890 \mathrm{~km}$ along its cycloid trajectory, where $d_{\mathrm{L}}\left(\mathrm{O}_{\mathrm{PUI}}^{+}\right)=8 r_{\mathrm{L}}\left(\mathrm{O}_{\mathrm{PUI}}^{+}\right)$is the length of one full gyrocycloid. For $\mathrm{O}_{2, \mathrm{PUI}}^{+}$multiply these values by 2 and for $\mathrm{H}_{\mathrm{PUI}}^{+}$divide by 16 .

How do these spatial scales compare with the spacecraft orbits? MAVEN detected the plume below the altitude of $\sim 3,000 \mathrm{~km}$ and MEX below the altitude of $\sim 2,000 \mathrm{~km}$ along the analyzed orbits. The apoapses of the orbits are $6,400 \mathrm{~km}$ for MAVEN and 10,500 km for MEX. Thus, $\mathrm{O}^{+}$and $\mathrm{O}_{2}^{+}$ions moving in the undisturbed upstream solar wind would require much longer distances than the MAVEN and MEX apoapses to complete their first half of the gyroperiod. However, protons can undergo several gyrations within these altitude ranges.

In principle the spacecraft could detect planetward oxygen ions at 2,000-3,000 km altitudes that have been ionized higher up in the exosphere and would have already completed the first half or even several gyroperiods. These would be planetary ions moving from the $-\mathrm{E}_{\mathrm{sw}}$ hemisphere toward the $+\mathrm{E}_{\mathrm{sw}}$ hemisphere and some of them would precipitate in the ionosphere ("the planetward heavy ion plume") (Curry, Luhmann, Ma, Liemohn, et al., 2015; Hara et al., 2013; Leblanc et al., 2015; Luhmann et al., 1992). However, the density of this population would be very low compared to the ionospheric source forming the heavy ion plume on the $+\mathrm{E}_{\mathrm{sw}}$ hemisphere. 
According to the model, the strongest oxygen ion flux in the plume is seen with ions that have traveled $\mathrm{DoF}=200-3,000 \mathrm{~km}$ in time of ToF $=20-80 \mathrm{~s}$ after ionization and have reached the energies of about $100 \mathrm{eV}-1 \mathrm{keV}$. In the periapsis column of Figure 4 intense populations of low energy $(10-100 \mathrm{eV})$ oxygen ions with long flight times ( $\mathrm{ToF}>100 \mathrm{~s}$ ) and distances ( $\mathrm{DoF}=1,000-10,000 \mathrm{~km}$ ) are seen. These populations appear to be nonmonotonous with respect to energy and ToF and DoF or at least have less efficient acceleration compared to pure ion pickup in the upstream. Long lifetimes at low energies are consistent with high planetary ion densities at low altitudes, which can lead to shielding from the large-scale convection electric field of the solar wind (e.g., Jarvinen et al., 2009). More efficient acceleration of heavy ions occurs when particles from this seed population emitted from the ionosphere reach higher altitudes in the induced magnetoshere as seen in the plume column of Figure 4.

\subsection{Limitations}

The following limitations should be noted. All ions of each species are collected from grid cells of the model that touch the spacecraft orbits. That is, no instrument geometrical factors or field of view effects are considered but the analyzed model spectra are omnidirectional. Including the observational factors would remove some of the ion counts in Figures 3-5. Further, the MAVEN orbit passes inside the inner boundary of the model between 13:12 and 13:27 UT, which is seen as a minimum in the magnitude of the magnetic field in Figure $2 \mathrm{~d}$. No Mars magnetic crustal anomalies are included in the analyzed simulations runs.

The DoF and ToF measure the time and distance from an ionization point to an observation point as a function of particle energy. For the ionospheric populations "the ionization" corresponds to the emission of an ion from the ionosphere toward the induced magnetosphere as implemented at the inner boundary of the model. In reality most of the ionospheric ions may have been ionized deep in the ionosphere before their departure (emission) toward the induced magnetosphere. Thus, the modeled DoF and ToF should be interpreted as lower limits for the ionospheric populations. For the exospheric populations this is not an issue as the their ionization occurs above the inner boundary of the simulation.

In this work finite resistivity is used to add diffusion in the propagation of the magnetic field as described in the model section. The ionospheric obstacle is approximated as a superconducting sphere and, thus, not allowing any diffusion of the magnetic field in the ionosphere. However, allowing a finite resistivity within the obstacle, or other structured (e.g., layered) resistivity profiles, could be implemented to investigate cases when the ionospheres of unmagnetized planets become magnetized, for example, under enhanced dynamic pressure of the solar wind (Luhmann et al., 1980). Further, three-dimensional resistivity profiles have been used to study the surface conductivity of a terrestrial planet in a global hybrid model (Janhunen \& Kallio, 2004).

Run 2 was used in the detailed analysis of ion spectra and energization. Difference between the two performed simulation runs is the upstream IMF components and the solar wind density (Table 1). The structure of the Mars-induced magnetosphere is quite similar between the two runs as the IMF cone angle and magnitude is almost the same. The IMF clock angle differs by $48^{\circ}$ between the runs. This means that the induced magnetosphere rotated in the direction perpendicular to incident solar wind flow between the MAVEN inbound and outbound BS crossings. The effects of IMF rotations on the Mars plasma environment can be studied in global models (Modolo et al., 2012). However, to implement such rotations accurately, magnetic field measurements are needed from the upstream of the BS in the same time when another spacecraft is sampling the induced magnetosphere. The solar wind density changes by $25 \%$ between the two runs, which can lead to the BS moving closer to the planet and the more compressed induced magnetosphere in Run 2 compared to Run 1.

The simulation runs analyzed here use the exospheric neutral profiles defined in the ISSI model challenge for comparative global modeling studies (Brain et al., 2010; Kallio et al., 2010; Modolo et al., 2016). The upstream conditions and the ionospheric emission rates were chosen specifically to this case study, which is the first attempt to compare multispacecraft observations from the Mars plasma environment to a global hybrid simulation. The role of the upper atmospheric variability over different time scales on the Mars plasma environment and atmospheric escape should be further studied in global models in light of new observations (Bhattacharyya et al., 2015; Bougher et al., 2015; Chaffin et al., 2014; Halekas, 2017; Krasnopolsky, 2002).

\subsection{Escape Rates}

The global escape rates from Mars $(\mathcal{E})$ in the analyzed simulation Run 2 are the following (with the escape rate divided by the injection rate in parentheses): $\mathcal{E}\left(\mathrm{O}^{+}\right)=1.7 \times 10^{25} \mathrm{~s}^{-1}(38 \%), \mathcal{E}\left(\mathrm{O}_{2}^{+}\right)=2.1 \times 10^{25} \mathrm{~s}^{-1}$ $(35 \%)$, and $\mathcal{E}\left(\mathrm{H}^{+}\right)=3.6 \times 10^{24} \mathrm{~s}^{-1}(98 \%)$. The rates were determined by subtracting the rate of ions impacting 
the inner boundary from the injection rate. Note that only the photoionization of the Martian exosphere is considered explicitly in this study as the focus is on the heavy ion energization in the induced magnetosphere. Other ionization processes affect both the rate and spatial distribution of planetary ion production at Mars. The escape rates are listed here for completeness.

\section{Summary}

We studied oxygen ion energization in the Mars-solar wind interaction by comparing particle and magnetic field observations on the Mars Express and MAVEN missions to a global hybrid simulation. We found that large-scale structures of the Martian-induced magnetosphere and plasma environment as well as the Mars heavy ion plume as seen by multispacecraft observations are reproduced by the model. Using the simulation, we estimated the dynamics of escaping oxygen ions by analyzing their distance and time of flight as a function of the gained kinetic energy along spacecraft trajectories. In the upstream region the heavy ion energization resembles single-particle solar wind ion pickup acceleration as expected, while within the induced magnetosphere the energization displays other features including the heavy ion plume from the ionosphere. Oxygen ions take up to $80 \mathrm{~s}$ and travel the distance of $20,000 \mathrm{~km}$ after their emission from the ionosphere toward the induced magnetosphere or photoionization from the neutral exosphere before they have reached energies of $10 \mathrm{keV}$ in the plume along the analyzed spacecraft orbits. Lower oxygen ion energies of $100 \mathrm{eV}$ are reached faster in 10-20 s over the distance of 100-200 km in the plume. Our finding suggests that oxygen ions are typically observed within the first half of their gyrophase if the spacecraft periapsis is on the hemisphere where the solar wind convection electric field points away from Mars.

\section{Acknowledgments}

The work of the lead author including the development of the RHybrid simulation platform was carried out during his Academy of Finland Postdoctoral Researcher project (Decision 257831). RJ thanks Arto Sandroos for invaluable help in the implementation of RHybrid on the Corsair simulation platform. The work of MAVEN's SWIA, STATIC, and magnetometer and MEX/ASPERA-3 instrument teams are acknowledged for providing high-quality observations. Figures $1 \mathrm{~d}-1 \mathrm{f}$ were created using the Vislt open source visualization tool (Childs et al., 2012). Figures $3 a, 3 c, 3 e$, and $3 g$ were created with the AMDA science analysis system (http://amda.cdpp.eu/) provided by the Centre de Donnees de la Physique des Plasmas (CDPP) supported by CNRS, CNES, Observatoire de Paris, and Universite Paul Sabatier, Toulouse. Global hybrid simulations were performed using the RHybrid simulation platform, which is available under an open source license by the Finnish Meteorological Institute (https://github.com/fmihpc/rhybrid). The simulation data that support the findings of this study are available from the corresponding author upon reasonable request. MAVEN and Mars Express data are openly available on the NASA's Planetary Data System (https://pds.nasa.gov/) and the ESA's Planetary Science Archive (https://www.cosmos.esa.int/web/psa).

\section{References}

Barabash, S., \& Lundin, R. (2006). ASPERA-3 on Mars Express. Icarus, 182, 301 -307. https://doi.org/10.1016/j.icarus.2006.02.015

Barabash, S., Fedorov, A., Lundin, R., \& Sauvaud, J.-A. (2007). Martian atmospheric erosion rates. Science, 315, $501-503$. https://doi.org/10.1126/science.1134358

Bhattacharyya, D., Clarke, J. T., Bertaux, J.-L., Chaufray, J.-Y., \& Mayyasi, M. (2015). A strong seasonal dependence in the Martian hydrogen exosphere. Geophysical Research Letters, 42, 8678-8685. https://doi.org/10.1002/2015GL065804

Boman, E. G., Catalyurek, U. V., Chevalier, C., \& Devine, K. D. (2012). The Zoltan and Isorropia parallel toolkits for combinatorial scientific computing: Partitioning, ordering, and coloring. Scientific Programming, 20(2), 129-150.

Bougher, S. W., Pawlowski, D., Bell, J. M., Nelli, S., McDunn, T., Murphy, J. R., .. Ridley, A. (2015). Mars Global lonosphere-Thermosphere Model: Solar cycle, seasonal, and diurnal variations of the Mars upper atmosphere. Journal of Geophysical Research: Planets, 120, 311-342. https://doi.org/10.1002/2014JE004715

Brain, D., Barabash, S., Boesswetter, A., Bougher, S., Brecht, S., Chanteur, G., .. Terada, N. (2010). A comparison of global models for the solar wind interaction with Mars. Icarus, 206, 139-151. https://doi.org/10.1016/j.icarus.2009.06.030

Brain, D. A., McFadden, J. P., Halekas, J. S., Connerney, J. E. P., Bougher, S. W., Curry, S., .. Seki, K. (2015). The spatial distribution of planetary ion fluxes near Mars observed by MAVEN. Geophysical Research Letters, 42, 9142-9148. https://doi.org/10.1002/2015GL065293

Brecht, S. H. (1990). Magnetic asymmetries of unmagnetized planets. Geophysical Research Letters, 17, $1243-1246$. https://doi.org/10.1029/GL017i009p01243

Chaffin, M. S., Chaufray, J.-Y., Stewart, I., Montmessin, F., Schneider, N. M., \& Bertaux, J.-L. (2014). Unexpected variability of Martian hydrogen escape. Geophysical Research Letters, 41, 314-320. https://doi.org/10.1002/2013GL058578

Childs, H., Brugger, E., Whitlock, B., Meredith, J., Ahern, S., \& Pugmire, D. (2012). Vislt: An end-user tool for visualizing and analyzing very large data. In High performance visualization: Enabling extreme-scale scientific insight (pp. 357-372). Boca Raton, FL: CRC Press.

Connerney, J. E. P., Espley, J., Lawton, P., Murphy, S., Odom, J., Oliversen, R., \& Sheppard, D. (2015). The MAVEN magnetic field investigation. Space Science Reviews, 195, 257-291. https://doi.org/10.1007/s11214-015-0169-4

Curry, S. M., Luhmann, J. G., Ma, Y. J., Dong, C. F., Brain, D., Leblanc, F., ... Jakosky, B. (2015). Response of Mars O ${ }^{+}$pickup ions to the 8 March 2015 ICME: Inferences from MAVEN data-based models. Geophysical Research Letters, 42, 9095-9102. https://doi.org/10.1002/2015GL065304

Curry, S. M., Luhmann, J., Ma, Y., Liemohn, M., Dong, C., \& Hara, T. (2015). Comparative pick-up ion distributions at Mars and Venus: Consequences for atmospheric deposition and escape. Planetary and Space Science, 115, 35-47. https://doi.org/ 10.1016/j.pss.2015.03.026

Dai, W., \& Woodward, P. R. (1998). On the divergence-free condition and conservation laws in numerical simulations for supersonic magnetohydrodynamic flows. Astrophysical Journal, 494, 317-335. https://doi.org/10.1086/305176

Dong, Y., Fang, X., Brain, D. A., McFadden, J. P., Halekas, J. S., Connerney, J. E., ... Jakosky, B. M. (2015). Strong plume fluxes at Mars observed by MAVEN: An important planetary ion escape channel. Geophysical Research Letters, 42, 8942-8950. https://doi.org/10.1002/2015GL065346

Dubinin, E., Chanteur, G., Fraenz, M., Modolo, R., Woch, J., Roussos, E., ... Winningham, J. D. (2008). Asymmetry of plasma fluxes at Mars. ASPERA-3 observations and hybrid simulations. Planetary and Space Science, 56, 832-835. https://doi.org/10.1016/j.pss.2007.12.006

Fedorov, A., Budnik, E., Sauvaud, J.-A., Mazelle, C., Barabash, S., Lundin, R., ... Dierker, C. (2006). Structure of the martian wake. Icarus, 182, 329-336. https://doi.org/10.1016/j.icarus.2005.09.021

Halekas, J. S. (2017). Seasonal variability of the hydrogen exosphere of Mars. Journal of Geophysical Research, 122, $901-911$. https://doi.org/10.1002/2017JE005306

Halekas, J. S., Taylor, E. R., Dalton, G., Johnson, G., Curtis, D. W., McFadden, J. P., ... Jakosky, B. M. (2015). The solar wind ion analyzer for MAVEN. Space Science Reviews, 195, 125-151. https://doi.org/10.1007/s11214-013-0029-z 
Hara, T., Seki, K., Futaana, Y., Yamauchi, M., Barabash, S., Fedorov, A. O., ... Delcourt, D. C. (2013). Statistical properties of planetary heavy-ion precipitations toward the Martian ionosphere obtained from Mars Express. Journal of Geophysical Research: Space Physics, 118, 5348-5357. https://doi.org/10.1002/jgra.50494

Hietala, H., Sandroos, A., \& Vainio, R. (2012). Particle acceleration in shock-shock interaction: Model to data comparison. The Astrophysical Journal Letters, 751, L14. https://doi.org/10.1088/2041-8205/751/1/L14

Jakosky, B. M., Grebowsky, J. M., Luhmann, J. G., Connerney, J., Eparvier, F., Ergun, R., ... Yelle, R. (2015). MAVEN observations of the response of Mars to an interplanetary coronal mass ejection. Science, 350, 0210. https://doi.org/10.1126/science.aad0210

Janhunen, P., \& Kallio, E. (2004). Surface conductivity of Mercury provides current closure and may affect magnetospheric symmetry. Annales Geophysicae, 22, 1829-1837.

Jarvinen, R., \& Kallio, E. (2014). Energization of planetary pickup ions in the solar system. Journal of Geophysical Research: Planets, 119, 219-236. https://doi.org/10.1002/2013JE004534

Jarvinen, R., Kallio, E., Janhunen, P., Barabash, S., Zhang, T. L., Pohjola, V., \& Sillanpää, I. (2009). Oxygen ion escape from Venus in a global hybrid simulation: Role of the ionospheric $\mathrm{O}^{+}$ions. Annales Geophysicae, 27, 4333-4348.

Jarvinen, R., \& Sandroos, A. (2013). Solar wind interaction with Venus and Mars in a parallel hybrid code (Vol. 15). Vienna, Austria: EGU General Assembly Conference Abstracts.

Jarvinen, R., Brain, D. A., \& Luhmann, J. G. (2016). Dynamics of planetary ions in the induced magnetospheres of Venus and Mars. Planetary and Space Science, 127, 1-14. https://doi.org/10.1016/j.pss.2015.08.012

Kallio, E. (2001). Escaping of planetary ions from Mars and Venus. Advances in Space Research, 27, 1815-1824. https://doi.org/10.1016/S0273-1177(01)00330-1

Kallio, E., \& Janhunen, P. (2003). Modelling the solar wind interaction with Mercury by a quasi-neutral hybrid model. Annales Geophysicae, 21, 2133-2145.

Kallio, E., Jarvinen, R., \& Janhunen, P. (2006). Venus solar wind interaction: Asymmetries and the escape of $\mathrm{O}^{+}$ions. Planetary and Space Science, 54, 1472-1481. https://doi.org/10.1016/j.pss.2006.04.030

Kallio, E., Liu, K., Jarvinen, R., Pohjola, V., \& Janhunen, P. (2010). Oxygen ion escape at Mars in a hybrid model: High energy and low energy ions. Icarus, 206, 152-163. https://doi.org/10.1016/j.icarus.2009.05.015

Koskinen, H. E. J. (2011). Physics of space storms: From the solar surface to the Earth. Chichester: Springer.

Krasnopolsky, V. A. (2002). Mars' upper atmosphere and ionosphere at low, medium, and high solar activities: Implications for evolution of water. Journal of Geophysical Research, 107, 5128. https://doi.org/10.1029/2001JE001809

Leblanc, F., Modolo, R., Curry, S., Luhmann, J., Lillis, R., Chaufray, J. Y., ... Jakosky, B. (2015). Mars heavy ion precipitating flux as measured by Mars Atmosphere and Volatile EvolutioN. Geophysical Research Letters, 42, 9135-9141. https://doi.org/10.1002/2015GL066170

Ledvina, S. A., Brecht, S. H., Brain, D. A., \& Jakosky, B. M. (2017). Ion escape rates from Mars: Results from hybrid simulations compared to MAVEN observations. Journal of Geophysical Research: Space Physics, 122, 8391 -8408. https://doi.org/10.1002/2016JA023521

Luhmann, J. G., Elphic, R. C., Russell, C. T., Mihalov, J. D., \& Wolfe, J. H. (1980). Observations of large scale steady magnetic fields in the dayside Venus ionosphere. Geophysical Research Letters, 7, 917-920. https://doi.org/10.1029/GL007i011p00917

Luhmann, J. G., Johnson, R. E., \& Zhang, M. H. G. (1992). Evolutionary impact of sputtering of the Martian atmosphere by O+ pickup ions. Geophysical Research Letters, 19, 2151-2154. https://doi.org/10.1029/92GL02485

Lundin, R. (2011). Ion acceleration and outflow from Mars and Venus: An overview. Space Science Reviews, 162, $309-334$. https://doi.org/10.1007/s11214-011-9811-y

Ma, Y. J., Russell, C. T., Fang, X., Dong, Y., Nagy, A. F., Toth, G., ... Jakosky, B. M. (2015). MHD model results of solar wind interaction with Mars and comparison with MAVEN plasma observations. Geophysical Research Letters, 42, 9113-9120. https://doi.org/10.1002/2015GL065218

Matsunaga, K., Seki, K., Brain, D. A., Hara, T., Masunaga, K., Mcfadden, J. P., ... Jakosky, B. M. (2017). Statistical study of relations between the induced magnetosphere, ion composition, and pressure balance boundaries around Mars based on MAVEN observations. Journal of Geophysical Research: Space Physics, 122, 9723-9737. https://doi.org/10.1002/2017JA024217

McFadden, J. P., Kortmann, O., Curtis, D., Dalton, G., Johnson, G., Abiad, R., ... Jakosky, B. (2015). MAVEN SupraThermal and Thermal lon Compostion (STATIC) instrument. Space Science Reviews, 195, 199-256. https://doi.org/10.1007/s11214-015-0175-6

Modolo, R., Chanteur, G. M., \& Dubinin, E. (2012). Dynamic Martian magnetosphere: Transient twist induced by a rotation of the IMF. Geophysical Research Letters, 39, L01106. https://doi.org/10.1029/2011GL049895

Modolo, R., Hess, S., Mancini, M., Leblanc, F., Chaufray, J.-Y., Brain, D., ... Mazelle, C. (2016). Mars-solar wind interaction: LatHyS, an improved parallel 3-D multispecies hybrid model. Journal of Geophysical Research: Space Physics, 121, 6378-6399. https://doi.org/10.1002/2015JA022324

Najib, D., Nagy, A. F., Tóth, G., \& Ma, Y. (2011). Three-dimensional, multifluid, high spatial resolution MHD model studies of the solar wind interaction with Mars. Journal of Geophysical Research, 116, A05204. https://doi.org/10.1029/2010JA016272

Nilsson, H., Edberg, N. J. T., Stenberg, G., Barabash, S., Holmström, M., Futaana, Y., .. Fedorov, A. (2011). Heavy ion escape from Mars, influence from solar wind conditions and crustal magnetic fields. Icarus, 215, 475-484. https://doi.org/10.1016/j.icarus.2011.08.003

Pohjola, V., \& Kallio, E. (2010). On the modeling of planetary plasma environments by a fully kinetic electromagnetic global model HYB-em. Annales Geophysicae, 28, 743-751. https://doi.org/10.5194/angeo-28-743-2010

Sandroos, A. (2013). CORSAIR solar energetic particle model. (AGU Spring Meeting Abstracts SH53B-01), American Geophysical Union.

Shimazu, H. (1999). Three-dimensional hybrid simulation of magnetized plasma flow around an obstacle. Earth Planets Space, 51, $383-393$. 